\title{
Generation of unipolar pulses from nonunipolar optical pulses in a nonlinear medium
}

\author{
Victor V. Kozlov, ${ }^{1,2}$ Nikolay N. Rosanov, ${ }^{3,4}$ Costantino De Angelis, ${ }^{1}$ and Stefan Wabnitz ${ }^{1}$ \\ ${ }^{1}$ Department of Information Engineering, Università degli Studi di Brescia, Via Branze 38, I-25123 Brescia, Italy \\ ${ }^{2}$ Department of Physics, St. Petersburg State University, Petrodvoretz, St. Petersburg, 198504, Russia \\ ${ }^{3}$ Institute of Laser Physics, Vavilov State Optical Institute, Birzhevaya liniya, 12, St. Petersburg, 199034, Russia \\ ${ }^{4}$ St. Petersburg State University of Information Technologies, Mechanics and Optics, St. Petersburg, 197101, Russia
}

(Received 15 December 2010; revised manuscript received 10 June 2011; published 12 August 2011)

\begin{abstract}
A unipolar electromagnetic pulse is a pulse with nonzero value of the static component of the Fourier spectrum of its real electric field (and not its envelope). We show how to efficiently generate unipolar pulses through propagation of an initially nonunipolar pulse in a nonlinear optical medium. One of the major results is the demonstration that the static component can only be generated in equal portions between the forward- and backward-traveling waves in the presence of nonlinear backscattering in a nonlinear medium.
\end{abstract}

DOI: $10.1103 /$ PhysRevA.84.023818

PACS number(s): 42.65.Ky, 41.20.Jb, 42.65.Re

\section{INTRODUCTION}

A sequence of intense unipolar electromagnetic pulses may coherently accelerate electrons in a plasma, as proposed in Ref. [1]. This publication was followed by an intense discussion on the possible applications of nonunipolar pulses: see for instance in Refs. [2-4], to mention just a few. In the literature, many authors have expressed their strong doubts that unipolar pulses, even if they may exist as a formal solution to the Maxwell equations, can ever be generated by means of practically available sources. This paper is aimed at resolving this long-standing controversy by demonstrating, through approximation-free direct numerical solutions of Maxwell's equations, that the generation of unipolar pulses is indeed possible by injecting in a nonlinear medium an initially nonunipolar pulse from a "conventional" laser source. By the term conventional we define the source which delivers nonunipolar pulses.

\section{PHYSICAL CONSIDERATIONS}

Unipolar electromagnetic pulses are understood here in a broad sense, namely as pulses with nonzero value of the electric field area

$$
A_{E}(z) \equiv \int_{-\infty}^{+\infty} d t E(z, t),
$$

where $E$ is the electric field strength and $t$ time. By taking the Fourier transform of the electric field $E(z, t)$ as $\bar{E}(z, \omega)=$ $\int d t \exp (i \omega t) E(z, t)$ and comparing with Eq. (1), we can see that the condition of nonzero area means that the static Fourier component of the electric field is different from zero: $\bar{E}(z, \omega=0) \neq 0$. Here, we shall only consider for simplicity the one-dimensional geometry involving the longitudinal coordinate $z$ and reserve a comment on the full three-dimensional configuration till the end. The one-dimensional model can be justified by considering a pulse propagating in a nonlinear medium, which is enclosed into a waveguide without cut-off frequency, such as for instance a metallic coaxial waveguide.

Oftentimes in the literature on generation of few-cycle pulses a difference is made between the two following representations of the electric field: $E_{\sin }(t)=A(t) \sin \left(\omega_{0} t\right)$ and $E_{\mathrm{cos}}(t)=A(t) \cos \left(\omega_{0} t\right)$, where $A(t)$ is an envelope (not nec- essarily slowly varying), for instance described by Gaussian function, and $\omega_{0}$ a central frequency. For any time-symmetric envelope, the first representation yields zero electric area, while the second representation yields nonzero area. Most treatments of few-cycle pulses appear cautious of dealing with nonzero area pulses: typically it is explicitly or implicitly assumed that pulse tails [which are not included in the expression of $\left.E_{\mathrm{cos}}(t)=A(t) \cos \left(\omega_{0} t\right)\right]$ are present so that the static component of the pulse is exactly compensated to zero. Moreover, in many studies on few-cycle pulses an important assumption in the derivation of approximate equations of motion is the requirement that the electric field area is exactly equal to zero, without, however, laying convincing physical grounds for such supposition. The lack of a clear understanding of which physical situations permit one to safely suppose that $A_{E}=0$, as opposed to what circumstances may enable the emergence of a sizable static Fourier component of the electric field, is the motivation behind our study.

A common argument against the possibility of generating the static component is based on the following analysis of the one-dimensional wave equation in its general form:

$$
\left(c^{2} \partial_{z}^{2}-\partial_{t}^{2}\right) E(t, z)=4 \pi \partial_{t}^{2} P(t, z) .
$$

By taking the Fourier transform of the previous equation, one obtains that its right-hand side vanishes for $\omega=0$, so that $\partial_{z}^{2} E(\omega=0, z)=0$. As a result, the commonly accepted conclusion is that the static component of the electric field cannot be amplified (or absorbed) in the course of pulse propagation through any medium. Although being delusively straightforward, as we shall see in this paper, such simple conclusion turns out to be false. As a matter of fact, we will show that: (i) the static component is always generated in a nonlinear medium; (ii) at the same time such generation process is fully compatible with the wave equation.

Let us start by writing Maxwell's equations for the linearly polarized electric $E(z, t)$ and magnetic $H(z, t)$ fields incident from vacuum on a slab of a nonmagnetic medium $(H=B)$ without free charges and electric currents:

$$
\partial_{t} D+\partial_{z} H=0 \quad \text { and } \quad \partial_{t} H+\partial_{z} E=0,
$$

where the speed of light $c$ is set to unity. Here the displacement field $D$ accounts for the medium polarization $P: D=$ 


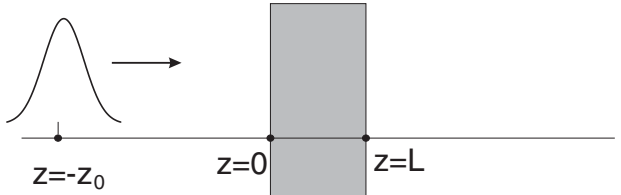

(a) $t=-T$

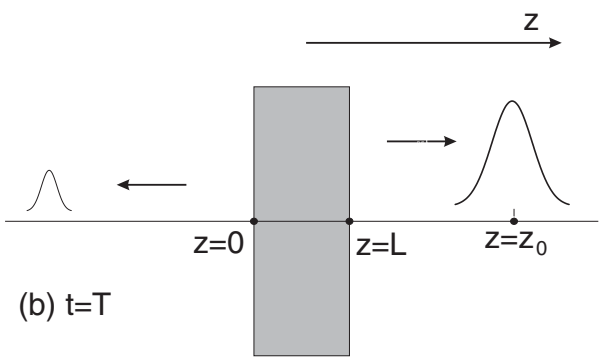

FIG. 1. Sketch of a typical optical experiment, showing the pulse incident from vacuum on the boundary with a medium: (a) before interaction $(t=-T \rightarrow-\infty)$; (b) after interaction $(t=T \rightarrow \infty)$. Throughout all simulations, we set the linear refractive index $n$ of the nonlinear medium equal to unity in order to avoid any linear Fresnel reflection. A similar goal can be achieved for $n$ different from unity, provided the nonlinear medium is surrounded by a linear medium with the same refractive index (index matching).

$E+4 \pi P$. Following Ref. [5], we may formulate the area theorem for the electric field in the form

$$
\frac{d}{d z} \int_{-\infty}^{+\infty} d t E(z, t)=0
$$

which is derived under the assumption that $E$ vanishes at $t \rightarrow \pm \infty$, i.e., the radiation is in the form of a time-localized pulse. It is rather common to draw from this equation the immediate conclusion that if $A_{E}$ is set to zero at $z=-z_{0}$, then it stays zero for all following points in space. The drawback of this conclusion is that $A_{E}\left(z=-z_{0}\right)$ is not a priori a known value, i.e., before the propagation problem is fully solved: in order to find $A_{E}\left(z=-z_{0}\right)$, we need to know the profile of $E$ from $t=-\infty$ to $+\infty$. By looking at the sketch in Fig. 1, we can see that the pulse passes through the point $-z_{0}$ twice: first, before the interaction, and then after. Even if the medium has no sharp boundaries, a back-reflected wave is always continuously generated while the pulse propagates through a nonlinear medium. Therefore, $A_{E}\left(-z_{0}\right)$ may only be known after fully solving the pulse propagation problem. The same conclusion applies at any other point in space. Strictly speaking, Eq. (4) cannot be considered as an evolution equation. This is because time $t$ and not space $z$ is the proper evolution variable for propagation problems based on Maxwell's equations. On the other hand, the area theorem

$$
\frac{d}{d t} \int_{-\infty}^{+\infty} d z H(z, t)=0
$$

which was derived in Ref. [6] for the magnetic field area $A_{H} \equiv$ $\int_{-\infty}^{+\infty} d z H(z, t)$, is indeed a proper evolution equation. On the basis of Eq. (5), we may predict that if before the interaction (at $t=-T$ ) the magnetic field area was zero, then it stays zero at all later times, that is during and after any interaction with a medium. Thus, for any electromagnetic pulse from a conventional laser source, we may always set $A_{H}=0$.

In this respect, we may note that the wave equation in the form $\partial_{z}^{2} E(\omega=0, z)=0$, discussed above, similarly to Eq. (4), is not a proper evolution equation. Therefore, the wave equation is unappropriate to describe the dynamics of the static component, as it yields the same conclusions as the area theorem in the form of Eq. (4).

\section{GENERATION OF THE STATIC COMPONENT IN A QUADRATIC MEDIUM}

The previous discussion shows that the only conclusion that can be derived from Eq. (4) is that at all points in space the static Fourier component of the electric field is equal to one and the same value. We are about to show that this value is always different from zero when a pulse interacts with a nonlinear medium. In particular, significant nonzero electric field area may be generated in a quadratic medium, i.e., whenever the nonlinear polarization reads as $P=P^{(2)}=\chi^{(2)} E^{2}$. As we are going to show next, the optical rectification process $(\omega-\omega)$ (which is known to lead to the generation of low-frequency radiation) introduces a zero-frequency component in the electric field of the reflected and transmitted pulses, however, not in a trivial way.

The finite difference time domain (FDTD) method was employed for integrating Maxwell's equations with the goal of solving the propagation problem shown in Fig. 2. In Fig. 2, we plot the input pulse shape $E_{\text {in }}$ as well as the pulse shapes after the pulse has experienced an interaction with a
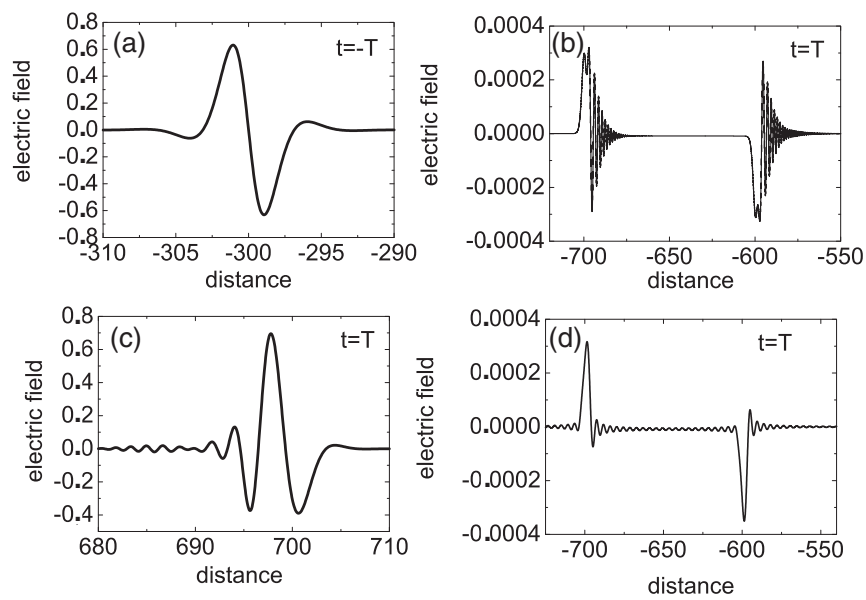

FIG. 2. Pulse shapes (a) before and (b-d) after interaction with the quadratic medium in the configuration shown in Fig. 1. The input shape is given by $E_{\text {sin }}(z, t=-T)=A(z) \sin \left(k_{0} z+\phi_{0}\right)$ with $A(z)=\operatorname{sech}\left[\left(z-z_{0}\right) / z_{p}\right]$. Here, $\omega_{0}=k_{0}=1\left(k_{0}\right.$ being the central wave number), $z_{p}=1.25, z_{0}=300, L=50,4 \pi \chi^{(2)} \max \left(E_{\text {in }}\right)=$ 0.01 , and $2 T=1000$. (b) Reflected shape; (c) transmitted shape; and (d) reflected shape after low bandpass frequency filter. Throughout all simulations, linear refractive index of the medium $n=1$. The frequency filter filters away frequencies above $1.5 \omega_{0} . E_{\text {in }}(z)$ is the profile of the incident field. The distance (time) is measured in physical units of $k_{0}\left(\omega_{0}\right)$. The field is normalized in such a way that $\max (E)=10^{-2} / \chi^{(2)}$. For the example with GaAs, this means that one unit is equal to $7000 \mathrm{~V} / \mathrm{m}$. 
quadratic medium with $4 \pi \chi^{(2)} \max \left(E_{\mathrm{in}}\right)=0.01$. Key to our analysis is the study of the reflected pulse, which is composed of two subpulses. The leading (subsequent) subpulse is the result of the reflection from the first boundary at $z=0$ (second boundary at $z=-L$ ). A distributed reflection from the interior of the medium takes place as well: its level is 50 times lower than the contribution from the boundaries, therefore it is not visible on the scale of the figure. Note that in vacuum temporal and spatial Fourier spectra coincide, therefore the unipolar character in space entails an unipolar property in time as well. The spectral content of each subpulse in Fig. 2(b) mainly consists of two contributions. Namely, low-frequency components and the second harmonic of the incident pulse. By filtering away all but the low-frequency components by means of a lowpass filter, we clearly observe in Fig. 2(d) the unipolar character of each subpulse. Our simulations show that the electric field area of the reflected portion of the input pulse computed at $z=-z_{0}$ is exactly equal to the electric field area of the transmitted pulse that is computed at $z=z_{0}+L$, namely $A_{E}=-0.000846$. Indeed, the computations confirm that in between these two points the area has strictly the same value, in full accordance with the area theorem in Eq. (4). Note that the areas of the two subpulses in reflection do not exactly compensate for each other, as it may look from visually inspecting Figs. 2(b) and 2(d). We also confirmed by direct calculation that the magnetic field area is conserved and stays equal to zero at all times, as dictated by Eq. (5); see Fig. 3. However, when we separated the total magnetic area in between reflected and transmitted waves, we observed that the reflected and transmitted portions introduced as $\left(A_{H}\right)_{\mathrm{ref}}=\int_{0}^{\infty} d z H$ and $\left(A_{H}\right)_{\mathrm{tr}}=\int_{-\infty}^{0} d z H$ are $\left(A_{H}\right)_{\mathrm{ref}}(t=$ $T)=-\left(A_{H}\right)_{\mathrm{tr}}(t=T)=0.000864$; see Fig. 3. Therefore, the static component is shared in equal portions between forwardand backward-traveling waves (with opposite signs for the

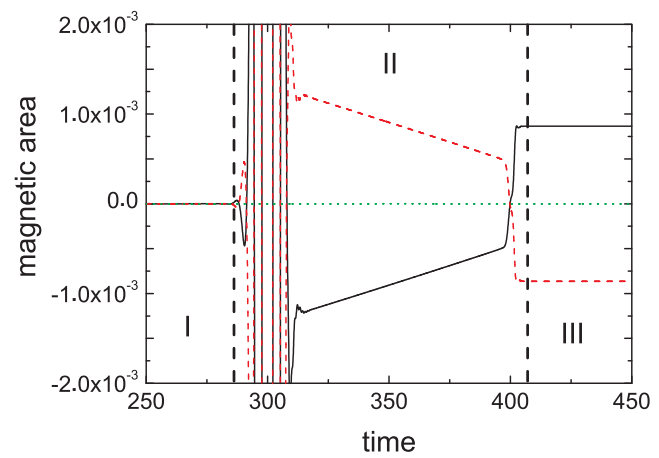

FIG. 3. (Color online) Evolution of the total magnetic area $A_{H}$ (dotted green), the magnetic area $\left(A_{H}\right)_{\text {ref }}$ of the reflected portion of the pulse (dashed red), and the magnetic area $\left(A_{H}\right)_{\text {tr }}$ of the transmitted portion of the pulse (solid black), as functions of time. Three stages are shown. Stage I (from $t=0$ till $t \approx 300$ ): propagation in vacuum prior to the interaction with the nonlinear medium. Stage II (from $t \approx 300$ till $t \approx 400$, the total interaction time with the nonlinear medium of the forward- and backward-traveling waves being equal to $2 L / c$ ): propagation through the nonlinear medium. Stage III (from $t \approx 400$ further on): propagation of the forward- and backward-traveling waves in vacuum after the interaction has finished. Parameters are as described in the legend of Fig. 2. magnetic field and equal signs for the electric one), and it is only present owing to nonlinear backscattering.

It is important to note that neither the transmitted nor the reflected area in isolation defines the static component. Only the total area defines the static component, because the static component must be defined along the entire time axis (by the integral over $H$ from $-\infty$ to $+\infty$ in time). The decomposition into the transmitted and reflected areas is introduced merely for the illustration of the dynamics of the magnetic field. So, the static component of the magnetic field stays strictly zero at all times, while the static component of the electric field is not zero.

\section{DISCUSSION}

The latter observation deserves some elaboration. In a weakly nonlinear medium (understood here as a medium whose linear index of refraction is perturbed only slightly by the nonlinear interaction with the field) the electromagnetic field contains electric and magnetic fields in portions of nearly identical intensity. This means that the spectrum of the electric field approximately coincides with the spectrum of the magnetic field. As it follows from the magnetic area theorem, the value of the static component stays zero before, during, and after the interaction of the field with a medium. This conclusion is compatible with the observation that the partial magnetic areas $\left(A_{H}\right)_{\text {ref }}$ and $\left(A_{H}\right)_{\text {tr }}$ are only different from zero because of the flip of the sign of the magnetic field upon reflection from the boundary, i.e., $\left(A_{H}\right)_{\mathrm{tr}}=-\left(A_{H}\right)_{\mathrm{ref}}$. In contrast, the electric field does not flip its sign: as a result, the value of the static component of the electric field is nonzero on the entire $z$ axis. As long as the medium is only weakly nonlinear, we can write $A_{E} \approx\left(A_{H}\right)_{\mathrm{tr}}=-\left(A_{H}\right)_{\text {ref }}$.

The conservation of the total magnetic field area $A_{H}$ (it stays zero forever, if it is zero initially) implies that pulses with nonzero partial $\left(A_{H}\right)_{\text {tr }}$ and $\left(A_{H}\right)_{\text {ref }}$ magnetic areas can only be generated in pairs. The transmitted portion of this pair has nonzero $\left(A_{H}\right)_{\mathrm{tr}}$ and it propagates forward. Whereas the reflected portion has $\left(A_{H}\right)_{\text {ref }}=-\left(A_{H}\right)_{\text {tr }}$ and it propagates backwards. Therefore, in the unidirectional approximation of electromagnetic field propagation, with initial conditions set by a nonunipolar pulse, no static electric or magnetic component may ever appear. Note that the reflected wave is a necessary companion of any nonlinear interaction, even in the absence of sharp boundaries, since a continuous distributed reflection always takes place. As a consequence, we may conclude that all unidirectional field propagation theories suffer from the inaccurate description of the low-frequency part of the pulse spectrum.

In our simulations, we observed that the value of the electric field area grows larger when increasing the intensity of the incident pulse. Another method of increasing the value of the static Fourier component of the electric field is to separate the two reflected subpulses. Supposing, for example, a nonlinear medium optical thickness of $1 \mathrm{~cm}$, the subpulses are separated in time by $60 \mathrm{ps}$, which would permit to switch out one of the subpulses by means of a fast (electro-optical) modulator. Another way to spatially separate the two subpulses is by using oblique (as opposed to normal) incidence of the input pulse into the nonlinear medium. In this way the two reflected subpulses will travel along different spatial directions. 
It is possible to obtain some analytical insight into the problem of generating nonzero area electric pulses by performing a perturbation study assuming that nonlinearity is weak; see Appendix for detailed derivations. We solved the initial boundary value problem of Maxwell's equations for a short electromagnetic pulse incident on a slab of nonlinear medium. We applied the continuity condition for the electric and magnetic fields on both boundaries. As a representative example, we consider the reflected wave, calculated in the given field approximation. In this approximation, the polarization inside the medium is supposed to be a function of the incident field $E_{\text {in }}(z-c t)$ at the boundary, as opposed to the exact local field in the nonperturbative approach. The reflected pulse is simply

$$
E_{\mathrm{ref}}(z-t)=\pi[P(z-2 L-c t)-P(z-c t)]
$$

(see Appendix A), where $P=P\left(E_{\text {in }}\right)$ is an arbitrary nonlinear polarization. Here the two terms in the right-hand side arise from reflections at the two boundaries, similarly to what was observed in Fig. 2(b). As follows from Eq. (6), in the given field approximation the area of $E_{\text {ref }}$ appears to be zero. Therefore, in the previously shown numerical results for the case of a quadratic medium with $P=P^{(2)}$, the nonzero area was obtained as a result of the interaction beyond the given field approximation [indeed, the transmitted pulse shown in Fig. 2(c) is sizably distorted as compared to the input shape in Fig. 2(a)]. However, as expected, the area of each subpulse in Eq. (6), $\chi^{(2)} \int d t E_{\sin }^{2}(t)$, can be rather large. Actually, because $\sin ^{2}\left(\omega_{0} t\right)=\frac{1}{2}\left[1-\cos \left(2 \omega_{0} t\right)\right]$, the intensity of the static component in each subpulse is as large as the intensity of the second harmonic.

Equation (6) formally shows that the static component of the polarization appears as a source of the electric field. This observation stands in contrast with the common (and false) argument, based on the observation that the source of the field in the wave equation is the second time derivative of $P$, stating that the static component of $P$ cannot act as a source of the electric field. As a matter of fact, the correct approach entails representing the solution of the field in the form of a double integral. This eliminates the double differentiation and sets the bare $P$ as the proper source of the field, as expressed by Eq. (6).

Quite strikingly, we found that the generation of the static Fourier component of the electric field in a cubic medium (with $P^{(3)}=\chi^{(3)} E^{3}$ ) can also be very efficient, although in the given field approximation [Eq. (6)] its intensity is zero, even for each reflected subpulse. When using $4 \pi \chi^{(3)} \max \left(E_{\text {in }}^{2}\right)=0.01$, the simulation result shown in Fig. 4 reveals that the total electric field area in between points $\left(z_{0}+L\right)$ and $-z_{0}$ is $A_{E}=$ -0.0004 (when compared with the value of $A_{E}=-0.000846$ that was obtained for a quadratic medium under similar conditions). Figure 4 shows that the frequency filtered reflected subpulses clearly exhibit a unipolar character, although this is not as pronounced as in the quadratic medium.

For relatively narrow band pulses, the cascaded frequency conversion processes (or higher harmonic generation), which take place in a cubic medium, may only lead to odd harmonics of the carrier frequency of the incident pulse. So, the generation of the static component does not appear to be possible. On the other hand, when using ultra-broadband pulses (e.g., a
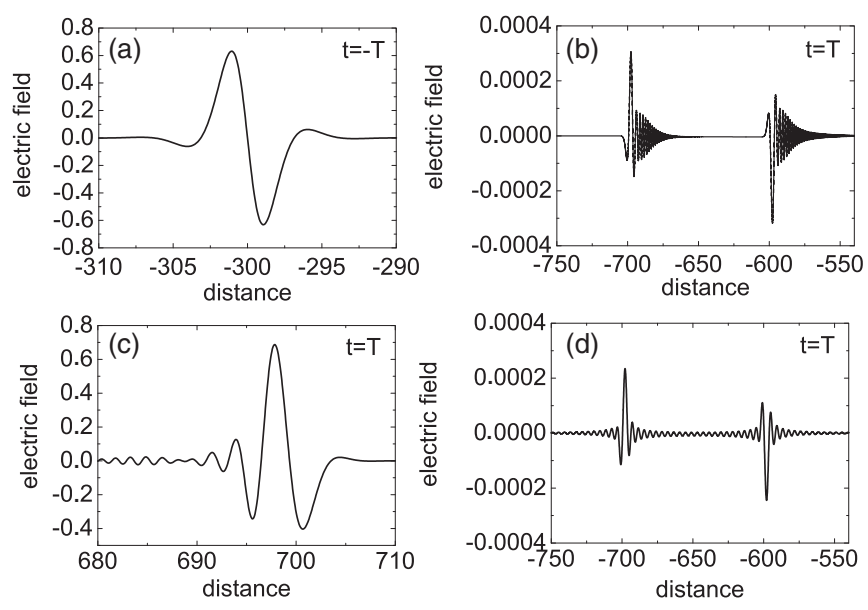

FIG. 4. Same as described in the legend of Fig. 2 but for the cubic medium, $P=\chi^{(3)} E^{3}$, with $4 \pi \chi^{(3)} \max \left(\left|E_{\text {in }}\right|^{2}\right)=0.01$.

1.25-cycle pulse in the example of Fig. 4, such pulses recently became available, see Ref. [7]), the nonlinear frequency conversion within the initial spectrum may lead to the generation of virtually any frequency, including the static component or zero frequency. In other words, the ultra-broadband nature of the incident pulse in our example explains the observed highly efficient generation of the static Fourier component; see Ref. [8].

In summary, our simulations have demonstrated the generation of the static Fourier component from an intense ultrashort pulse that does not contain this component and incident on either quadratic or cubic nonlinear media. As the nonlinearity of any nonlinear medium can be expanded in a series on $E$, thereby containing quadratic or cubic terms, or a combination of both, we may conclude that the generation of the static Fourier component of the electric field is a rather general feature of the nonlinear interaction of the electromagnetic field with nonlinear media. The intensity of the static Fourier component grows larger when increasing the efficiency of the frequency conversion process. Clearly, no new frequencies can be generated in a linear medium. Note that our definition of unipolar pulses via the requirement of the static component to have nonzero value is more rigorous than the plain observation of half-cycle bursts of radiation, which are followed by a tail that in some cases may even fully compensate the area of these bursts to zero. Moreover, according to this definition the unipolar pulses are not necessarily half-cycle pulses, as those observed, for instance, in Ref. [9]. As a matter of fact, the time duration of unipolar pulses can be as long as desirable.

\section{ABSORPTIVE AND DISPERSIVE NONLINEAR MEDIUM}

In the discussion presented above we were dealing with a minimal model, in which the medium exhibited only nonlinearity. However, in addition to its nonlinear properties, media can be absorptive and also dispersive. In this section, we shall study the efficiency of the generation of the static component in presence of absorption and dispersion. First, let us start with the absorption. 
Here, the absorption properties of the medium result from the interaction of the electric field with free carriers. From a practical point of view, this type of absorption is relevant to semiconductors interacting with electromagnetic fields with a carrier frequency below the bottom of the conduction band. In this situation, the absorption of light by bound electrons is inhibited, and only free electrons are able to absorb radiation. Then, the wave equation for the electric field reads

$$
\frac{\partial^{2} E}{\partial z^{2}}-\frac{4 \pi}{c} \frac{\partial j}{\partial t}-\frac{\partial^{2} D}{\partial t^{2}}=0 .
$$

In its turn, the electric current obeys the Drude equation:

$$
\frac{d j}{d t}+v j=\frac{\omega_{p}^{2}}{4 \pi} E .
$$

Here, $\omega_{p}$ is the plasma frequency, $v$ is the frequency of collisions of free electrons with ions and atoms. The model of conductivity that is supplied by the Drude equation [Eq. (8)] assumes the following form of the frequency-dependent conductivity:

$$
\sigma(\omega)=\frac{\omega_{p}^{2}}{4 \pi} \frac{1}{v-i \omega} .
$$

As estimated in Ref. [6] for GaAs, $v \approx 3 \mathrm{THz}$. Then, for an infrared field with the central frequency $\omega_{0}=300 \mathrm{THz}$, we get rather a rather steep dispersion of the conductivity: being $\sigma(0)=\omega_{p}^{2} / 4 \pi v$ for the static field, the conductivity drops down to $\sigma\left(\omega_{0}\right)=\omega_{p}^{2} \nu / 4 \pi \omega_{0}^{2}=10^{-4} \omega_{p}^{2} / 4 \pi \nu$ at the central frequency of the excitation field. Note that the condition of causality expressed by the (not shown but implied) KramersKronig relation, does not allow us to separate the absorption from dispersion. The two characteristics come hand in hand.

Figure 5(a) shows the value of the spectral power of the static component in a quadratic medium with the inclusion of the conductivity obtained by numerically simulating the previously described model. For relatively small values of the conductivity (i.e., $\left.4 \pi \sigma(0)<0.1 \omega_{0}\right)$, its influence on the generation of the static component is negligible. This range corresponds to semiconductors with moderate doping. For heavily doped semiconductors, the conductivity may rise up to $4 \pi \sigma(0) \sim 100 \omega_{0}$. As we can see from Fig. 5(a), with a conductivity in this range the power of the static component varies near the no-absorption value, with a minimum of about four orders of magnitude less than in the no-absorption case and with maximum of about two orders of magnitude larger than in the no-absorption case. Higher values of conductivity are quite rare for semiconductors and more characteristic to metals. In this metallic limit, the static component is generated rather inefficiently, even in reflection.

From the previously discussed results, it is quite difficult to conclude whether the nonmonotonic dependence of the spectral power of the static component should be attributed to the absorptive properties of the conductive medium or to its dispersive characteristics. In order to address this ambiguity, we performed computations with $v=100 \omega_{0}$, a value that is hardly achievable for optical fields but that gives us the possibility to study absorption in its pure form, i.e., without the admixture of dispersion. In this case the effects of dispersion are negligible when compared to absorptive effects.

In Fig. 5(b), we plot the same dependence as in Fig. 5(a) but with $v=100$ instead of $v=0.01$. The overall decay of the value of the static component takes place as a result of the reduction of the efficiency of the process of nonlinear frequency conversion. However, it is important to point out that the spectral power of the static component is related to the total reflected field, that is to simultaneously both subpulses in reflection. These subpulses are $\pi$ phase-shifted and therefore their contributions are subtracted from each other. If for some reason one of the subpulses becomes relatively smaller in intensity, then the overall efficiency of the generation of the static component is increased. This is exactly the case of severe absorption, when the subpulse coming from the second boundary is heavily absorbed, while the subpulse reflected from the first boundary is not. This compromise between the reduction of conversion efficiency leading to a reduction of the spectral power on the one hand and the strong attenuation of the second subpulse leading to the increase of the spectral power on the other hand, explains the appearance of humps in Fig. 5. Results shown in Fig. 5(a) are modified with respect to the results shown in Fig. 5(b) by the additional influence of dispersion.

Note that for very high values of conductivity, the principle of equality of the static components generated in forward- and backward-traveling waves no longer holds (red circles and
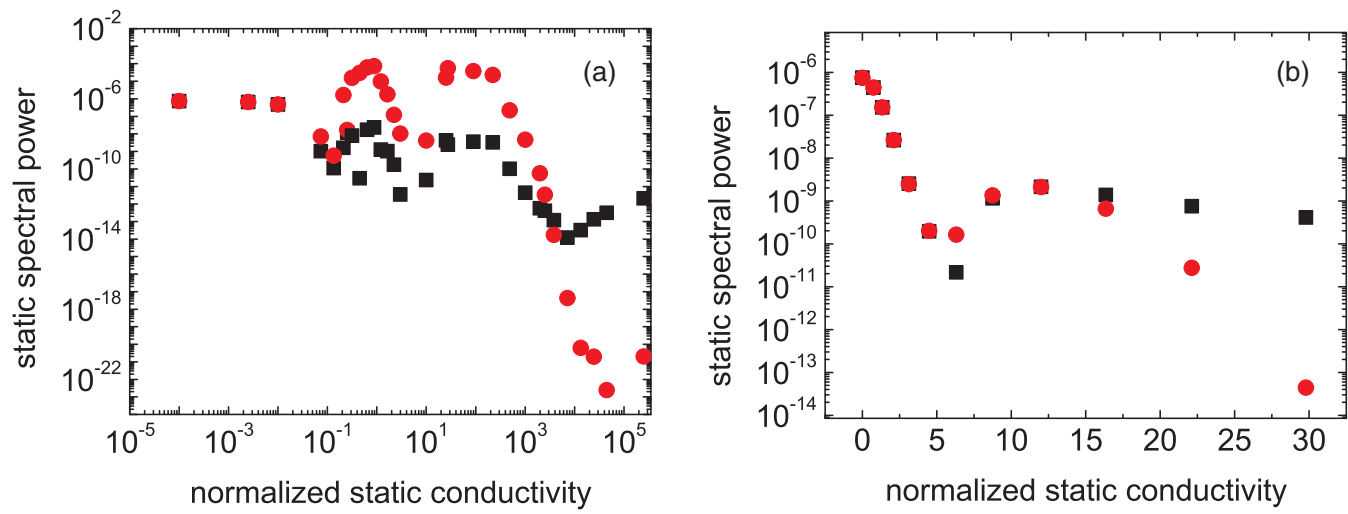

FIG. 5. (Color online) The spectral power of the static component in the quadratic medium with conductivity as a function of the conductivity normalized to the central frequency of the excitation field $\omega_{0}: 4 \pi \sigma(0) / \omega_{0}$. Red circles (black squares) indicate the value of the spectral power of the static component in the forward- (backward-)traveling wave. Other parameters are: $4 \pi \chi^{(2)} E_{\text {in }}=0.01$, (a) $v=0.01 \omega_{0}$; (b) $v=100 \omega_{0}$. 

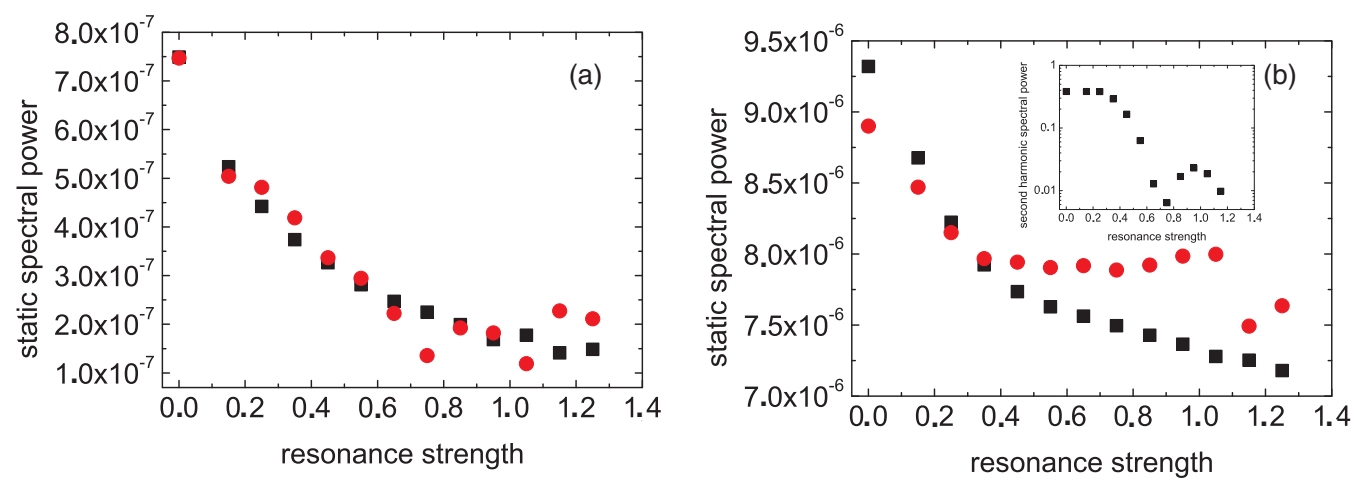

FIG. 6. (Color online) Spectral power of the static component in the quadratic medium with embedded two-level systems as a function of the resonance strength $\alpha$. Red circles (black squares) indicate the value of the spectral power of the static component in the forward(backward-)traveling wave. The inset shows the spectral power of the second harmonic of the forward-traveling wave for the same parameters as in the main figure. Parameters are: the resonance line is centered at $\omega_{\text {res }}=2.5 \omega_{0}$, relaxation time of polarization $\gamma_{\mathrm{ab}}=\gamma_{a} / 2=0.005 \omega_{0}$, where $\gamma_{a}$ is the relaxation time of the upper level, the lower level is the ground level. Other parameters are as in Fig. 2, except that for figure (b) the duration of the pulse is four times longer than in (a).

black squares do not coincide in Fig. 5). The reason is that the magnetic portion of the electromagnetic field no longer copies the shape of the electric field. Therefore, the conservation of the total magnetic area and its equality to zero does not give us any longer a hint of how the electric field behaves. Note that in this limit of high conductivity, optical fields decay so fast that nonlinearity has no chance to efficiently generate new harmonics. Such nonlinear media are impractical and are not used in optics. From this viewpoint, the principle of equality of the static components in the forward- and backward traveling waves is the principle which characterizes all practically relevant situations.

In Fig. 5(b), we consider the case for which absorption is greatly dominating over dispersion. It is instructive to consider the opposite limit. Such a dispersive limit arises, for instance, on a far-off-resonant wing of a resonant line of a two-level system. The absorption is also present in this case as demanded by Kramers-Kronig relations, but its magnitude is much smaller than the value of dispersion. As a specific model, we choose a quadratic medium with two-level systems embedded in it. The resonance is located at $\omega_{\text {res }}=2.5 \omega_{0}$, where $\omega_{0}$ is the central frequency of the excitation field. The dimensionless resonance strength defined as $\alpha=4 \pi N d^{2} / \hbar \omega_{0}$ determines the refractive index and the absorption coefficient. Here, $d$ is the dipole moment of the resonant transition and $N$ the concentration of the two-level systems. The steepness of the dispersion and absorption is regulated by the response function

$$
F(\omega)=\omega_{0} \frac{\gamma_{\mathrm{ab}}+i\left(\omega-\omega_{\mathrm{res}}\right)}{\left(\omega-\omega_{\mathrm{res}}\right)^{2}+\gamma_{\mathrm{ab}}^{2}},
$$

where $\gamma_{\mathrm{ab}}=0.005 \omega_{0}$ is the relaxation time of the polarization. For $\alpha=1$, the index of refraction at $\omega=\omega_{0}$ is $\approx(1+2 / 3)$, while at $\omega=0$ it is only $\approx(1+1 / 4)$. This dispersion is strong enough, so that we may cover a wide range of situations by scanning $\alpha$ from 0 to 1.3, as in Fig. 6. As we can see, the role of absorption is not crucial. Thus, in the process of transmission through a medium, the pulse only loses $10 \%$ of its energy for $\alpha=1.3$ in the case shown in Fig. 6(b).
The decaying character of the curves in Figs. 6(a) and 6(b) with the increase of the dispersion is attributed to the temporal spreading of the pulse. This spreading is accompanied by a drop in intensity, therefore a degradation of the frequency conversion results. The spreading and the drop in intensity is not so pronounced for longer pulses; see Fig. 6(b) in comparison with Fig. 6(a).

An important question is whether the process of generation of the static component needs to be phase-matched. Our answer is no. This conclusion is based on the analysis of Fig. 6 and its inset. We can see that the reduction of the spectral intensity of the static component is negligible in comparison with the two orders of magnitude decrease of the intensity of the second harmonic, whose generation in its turn requires phase-matching between the fundamental frequency and its second harmonic.

\section{CONCLUDING REMARKS}

We performed extensive simulations showing that the generation of the static component of the electric field in a dispersive medium is as efficient as in a dispersionless medium. Therefore, this process appears to be qualitatively different from any other harmonic generation process, in that it does not require obeying phase-matching conditions. In addition, our simulations also show that moderate levels of absorption do not influence the static component generation effect.

In physical units, our example of a quadratic medium would correspond to the case of a 12 fs pulse at the carrier wavelength of $3.31 \mu \mathrm{m}$ with a peak intensity of $10 \mathrm{MW} / \mathrm{cm}^{2}$ incident on a $26-\mu \mathrm{m}$-thick slab of GaAs with $\chi^{(2)}=138 \mathrm{pm} / \mathrm{V}$, [11]. Whenever the subpulses in reflection are not separated, the spectral power of the static component is $3 \times 10^{-7}$ lower than the peak spectral power at the fundamental frequency. On the other hand, if the subpulses are resolved, then the conversion efficiency into the static electric field component is 25 times larger. Experimentally, the low-frequency part of a reflected pulse (below $0.1 \mathrm{THz}$ ) can even be resolved by electronics, as it was done in the classical experiment on optical rectification; see Ref. [12]. 
So far, our analysis was restricted to the one-dimensional geometry, as we assumed the use of a waveguide configuration in order to combat diffraction. In free space, the dynamics of the unipolar pulses are essentially three-dimensional because of the short diffraction length which is characteristic to the lowfrequency components of the Fourier spectrum of a unipolar pulse. As a matter of fact, the diffraction length vanishes for the static component, which diffracts as a spherical wave. At the same time, the high-frequency portion of the spectrum diffracts at a substantially slower rate. As a consequence of the strong asymmetry in the strength of diffraction for lowfrequency components, on the one hand, and high-frequency components, on the other hand, the on-axis temporal shape of the unipolar pulse is severely distorted, as described for instance in Ref. [10]. Note, in this respect, that for an efficient generation of the static component the waveguide geometry is significantly advantageous over the free-propagation regime.

In conclusion, we have shown that unipolar electromagnetic pulses are generated in the course of the interaction of an intense broadband pulse with a weakly nonlinear medium. The static components of such unipolar pulses are equally intense in transmission and in reflection. In reflection, the unipolar character of the pulses is more pronounced, provided that the linear index of refraction of the nonlinear medium is equal to the index of the surrounding medium, so that there is no linear reflection. Therefore, existing theories based on assuming a zero value of the electric field area require modifications for properly taking into account the unavoidable generation of the static Fourier component of the electric field in virtually any nonlinear interaction with matter.

\section{ACKNOWLEDGMENT}

This work was carried out with support from the Cariplo Foundation Grant No. 2009-2730. N.N.R. acknowledges the Cariplo Foundation grant of Landau Network, Centro Volta for the support of his work at the Università degli Studi di Brescia, as well as Grant No. 2.1.1/9824 of the Russian Ministry of Education and Science and Grant No. 11-02-12250-ofi-m of the Russian Foundation for Basic Research. We thank an anonymous referee for his constructive remarks.

\section{APPENDIX: BOUNDARY-VALUE PROBLEM FOR A NONLINEAR SLAB}

Let us suppose that the electric field $E_{\text {in }}$ incident on a slab of a nonlinear medium is split into a reflected $E_{\text {ref }}$ and a transmitted $E_{\text {tr }}$ part. Thus, we may write (for the pulse incident on the first boundary at $z=0$ and passing through the second boundary at $z=L$ ),

$$
\begin{gathered}
E(z, t)=E_{\mathrm{in}}(z-t)+E_{\mathrm{ref}}(z+t), z<0 \\
E(z, t)=E_{\mathrm{tr}}(z-t), z>L .
\end{gathered}
$$

Assuming that the nonlinearity is relatively weak, we can develop a perturbation theory with the strength of the nonlinearity as small parameter: $E=E_{0}+E_{1}+E_{2}+\ldots$. In the zeroth order, $D=E_{0}$, therefore

$$
\frac{\partial^{2} E_{0}}{\partial z^{2}}-\frac{\partial^{2} E_{0}}{\partial t^{2}}=0
$$

The solution to this equation is the forward-traveling pulse $E_{0}=E_{\text {in }}(z-t)$, as dictated by the problem under consideration. In the first order, we write

$$
\frac{\partial^{2} E_{1}}{\partial z^{2}}-\frac{\partial^{2} E_{1}}{\partial t^{2}}=4 \pi \frac{\partial^{2}}{\partial t^{2}} P\left[E_{\text {in }}(z-t)\right],
$$

so that the right-hand side is a given function of $\xi=z-t$. We can write Eq. (A4) in terms of $\xi$ and $\eta=z+t$ as

$$
\frac{\partial^{2} E_{1}}{\partial \xi \partial \eta}=\pi \frac{\partial^{2}}{\partial \xi^{2}} P\left[E_{\text {in }}(\xi)\right] .
$$

The general solution to this equation reads

$$
E_{1}(\xi, \eta)=\pi \eta \frac{\partial P}{\partial \xi}+F(\xi)+B(\eta) .
$$

Here, the functions $F$ and $B$ are to be determined. If for the transmitted pulse we write $E_{\text {tr }}(\xi)=E_{\text {in }}(\xi)+\delta E_{\text {tr }}(\xi)$, then we get in terms of the original variables

$$
E_{1}(z, t)=\left\{\begin{array}{c}
E_{\mathrm{ref}}(z+t) \text { for } z<0, \\
\pi(z+t) P_{t}^{\prime}(z-t)+F(z-t)+B(z+t) \text { for } 0<z<L, \\
\delta E_{\mathrm{tr}}(z-t) \text { for } z>L .
\end{array}\right.
$$

Using the conditions that the electric field and its derivative are continuous on both boundaries we get four equations. Two equations hold for joining solutions at $z=0$ :

$$
\begin{gathered}
E_{\mathrm{ref}}(t)=\pi t P_{t}^{\prime}(-t)+F(-t)+B(t), \\
E_{\mathrm{ref}}^{\prime}(t)=\pi P_{t}^{\prime}(-t)+\pi t P_{t t}^{\prime \prime}(-t)+F_{t}^{\prime}(-t)+B_{t}^{\prime}(t),
\end{gathered}
$$

and two equations hold for joining solutions at $z=L$ :

$$
\delta E_{\mathrm{tr}}(L-t)=\pi(L+t) P_{t}^{\prime}(L-t)+F(L-t)+B(L+t),
$$

$$
\begin{aligned}
\delta E_{\mathrm{tr}}^{\prime}(L-t)= & \pi P_{t}^{\prime}(L-t)+\pi(L+t) P_{t t}^{\prime \prime}(L-t) \\
& +F_{t}^{\prime}(L-t)+B_{t}^{\prime}(L+t) .
\end{aligned}
$$

By differentiating Eq. (A8) by $t$ and combining with Eq. (A9) we obtain

$$
\begin{gathered}
E_{\mathrm{ref}}^{\prime}(t)=\pi P_{t}^{\prime}(-t)+B_{t}^{\prime}(t), \\
0=\pi t P_{t t}^{\prime \prime}(-t)+F_{t}^{\prime}(-t) .
\end{gathered}
$$


After changing $t$ to $-\xi$ Eq. (A13) yields

$$
F(\xi)=\pi \xi P_{\xi}^{\prime}(\xi)-\pi P(\xi)
$$

after integrating it once. In its turn, Eq. (A12) yields

$$
E_{\text {ref }}(t)=B(t)-\pi P(-t) .
$$

Similar manipulations with Eqs. (A10) and (A11) lead to relation

$$
B(t)=\pi P(2 L-t) .
$$

Combining Eqs. (A15) and (A16) we finally get

$$
E_{\mathrm{ref}}(t)=\pi[P(2 L-t)-P(-t)] .
$$

[1] B. Rau, T. Tajima, and H. Hojo, Phys. Rev. Lett. 78, 3310 (1997).

[2] K.-J. Kim, K. T. McDonald, G. V. Stupakov, and M. S. Zolotarev, Phys. Rev. Lett. 84, 3210 (2000).

[3] B. Rau, T. Tajima, and H. Hojo, Phys. Rev. Lett. 84, 3211 (2000).

[4] A. L. Troha and F. V. Hartemann, Phys. Rev. E 65, 028502 (2002).

[5] N. N. Rosanov, Optics Spectroscopy 107, 721 (2009).

[6] N. N. Rosanov, V. V. Kozlov, and S. Wabnitz, Phys. Rev. A 81, 043815 (2010).
[7] G. Krauss, S. Lohss, T. Hanke, A. Sell, S. Eggert, R. Huber, and A. Leitenstorfer, Nat. Photon. 4, 33 (2010).

[8] N. N. Rozanov, Optics Spectroscopy 104, 248 (2008).

[9] X. Song, W. Yang, Z. Zeng, R. Li, and Z. Xu, Phys. Rev. A 82, 053821 (2010).

[10] D. You and P. H. Bucksbaum, J. Opt. Soc. Am. B 14, 1651 (1997).

[11] P. S. Kuo, K. L. Vodopyanov, M. M. Fejer, D. M. Simanovskii, X. Yu, J. S. Harris, B. Bliss, and D. Weyburne, Opt. Lett. 31, 71 (2006).

[12] M. Bass, P. A. Franken, J. F. Ward, and G. Weinreich, Phys. Rev. Lett. 9, 446 (1962). 